SCIREA Journal of Information Science

and Systems Science

http://www.scirea.org/journal/ISSS

January 14, 2022

Volume 6, Issue 1, February 2022

https://doi.org/10.54647/isss12146

\title{
FILTERING A SIGNAL FROM NOISE AT A SINS COARSE INITIAL ALIGNMENT
}

\author{
A. Zbrutsky ${ }^{1}$, V.Meleshko ${ }^{2}$ \\ ${ }^{1}$ National technical university of Ukraine “Igor Sikorsky Kyiv Polytechnic Institute” , Kyiv, \\ 03056, Ukraine \\ ${ }^{2}$ National technical university of Ukraine “Igor Sikorsky Kyiv Polytechnic Institute” , Kyiv, \\ 03056, Ukraine \\ E-mail: zbrutsky@cisavd.kpi.ua (A. Zbrutsky),mvv44@ukr.net (V.Meleshko)
}

\begin{abstract}
The possibilities of using the Kalman filter for cleaning (filtering) signals from gy-roscopes and accelerometers of a strapdown inertial navigation system (SINS) with a rough initial alighnment on a fixed base are considered. For this, algorithms for averaging filters with forward and backward computations (forward and backward filters) are proposed. It is shown that with a relatively short recording of the signal $(30 \mathrm{~s})$, in comparison with the simple average of the signal, it is possible to signifi-cantly, practically to zero, reduce the influence of the measurement noise. This is achieved by successively applying forward and then backward filters. The results were obtained both in the modeling of filtration and in the field experiment of the initial alighnment.
\end{abstract}

Keywords: strapdown inertial navigation system (SINS), Coarse initial alighnment , Kalman filter 


\section{Introduction}

A rough initial alignment of a strapdown inertial navigation system (SINS) is most often performed on a stationary base, when the signals of the gyroscopes and accelerometers can be taken stationary. The alignment is called rough conventionally, so later, clarification procedures can be performed, called the precision alignment.

The initial alignment mode is characterized by two main parameters - accuracy and time. The accuracy depends on the alignment algorithm (precision or iterative $[1,2,3]$ ), systematic and random errors (noise) of gyroscopes and accelerometers. The main problem of rough alignment is the selection of the useful signal component in very high noise conditions. The noise can be tens of times greater than the useful signal. Figure 1 shows the signals of laser gyroscopes (axis $\mathrm{y}-\mathrm{deg} / \mathrm{hr}$ ) and accelerometers (axis $\mathrm{y}-\mathrm{m} / \mathrm{s}^{2}$ ) and their characteristics (mean value, std - root-mean-square deviation).

To filter the signal from the noise, a sufficiently long (tens of minutes) recording of the signal is required. At the same time, the working conditions of an object with SINS may require an alignment in a time not exceeding $1 \mathrm{~min}$. Thus, there is always a contradiction between the accuracy and the time of the alignment. These characteristics need to be explored and brought together.

To filter signals, simple averaging, moving average, exponential average, algorithms based on the least squares method, various modifications of Kalman filters [2,4,5], and wavelets $[6,7,8,9]$ are used.

\section{Problem statement}

Let's consider the application of the Kalman filter for filtering the signals of the SINS sensors. We will obtain averaging Kalman filters with forward flow [2] and with backward flow calculations, based on the provisions set forth in $[10,11,12,13,14]$. Will represent the linear model of the system under study in matrix form

$$
\overrightarrow{\dot{x}}=\mathbf{A} \overrightarrow{\mathbf{x}}+\mathbf{B} \overrightarrow{\mathrm{u}}+\mathbf{G} \overrightarrow{\mathrm{w}},
$$

where $\overrightarrow{\mathrm{x}}$ is the n-dimensional vector of the systems state; $\overrightarrow{\mathrm{u}}$ - $\mathrm{r}$-dimensional control vector; $\overrightarrow{\mathrm{W}}$ - k-dimensional vector of random actions; $\mathbf{A}$ - matrix of state with nxn dimension; $\mathbf{B}$ is a control matrix with nxr dimension; $\mathbf{G}$ - matrix of transfer the random actions with nxk dimension.

Usually the observer can't measure the full vector of the systems state. Measured information (available or necessary) is determined by the equation 

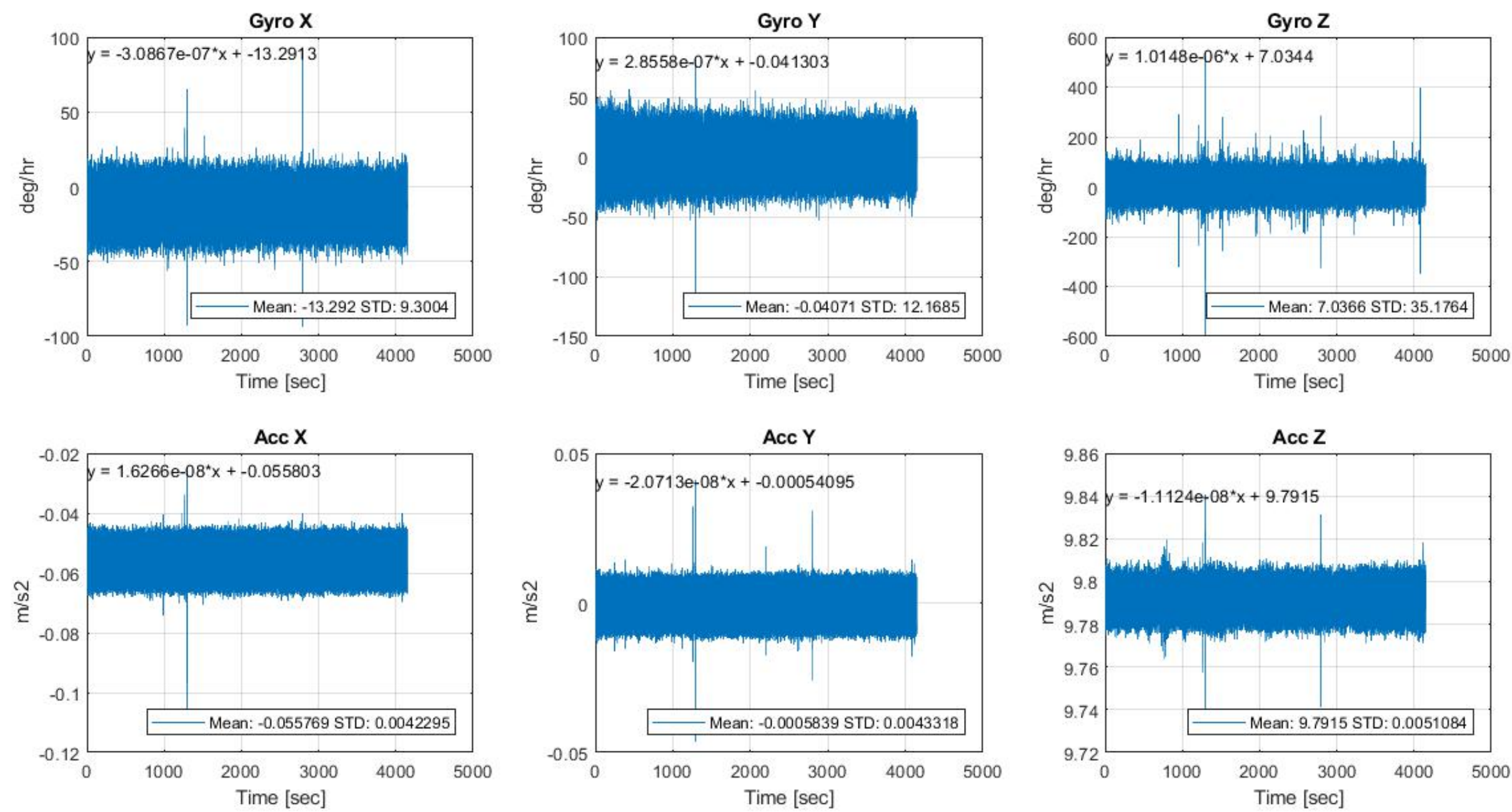

Figer1. Signals from SINS gyroscopes and accelerometers on a fixed base 


$$
\vec{y}=\mathbf{H} \vec{x}+\vec{v}
$$

where $\vec{y}-m$-dimensional vector of measurement; $\vec{v}-m$ - dimensional vector of measurement nois; $\mathbf{H}$ - is a matrix of measurement with mxn dimension.

Influences $\mathbf{w}$ and noise $\mathbf{v}$ will be considered Gaussian random processes of the white noise type with zero mathematical expectation

$$
\mathrm{M}\left[\mathrm{w}(\mathrm{t}) \mathrm{w}^{\mathrm{T}}(\tau)\right]=\mathbf{Q}(\mathrm{t}) \delta(\mathrm{t}-\tau) ; \quad \mathrm{M}\left[\mathrm{v}(\mathrm{t}) \mathrm{v}^{\mathrm{T}}(\tau)\right]=\mathbf{R}(\mathrm{t}) \delta(\mathrm{t}-\tau),
$$

where $\mathbf{Q}(\mathrm{t})$ - symmetric nonnegative definite $(\mathrm{k} \times \mathrm{k})$ white noise intensity matrix $\mathrm{w}(\mathrm{t}) ; \mathbf{R}(\mathrm{t})$ symmetric positive definite $(\mathrm{m} \times \mathrm{m})$ - white noise intensity matrix $\mathrm{v}(\mathrm{t}) ; \delta(\mathrm{t})$ - delta - Dirac function.

Initial state of the system $\mathrm{x}\left(\mathrm{t}_{0}\right)$ characterized by a known mathematical expectation $\mathrm{M}\left[\mathrm{x}\left(\mathrm{t}_{0}\right)\right]=\overline{\mathrm{x}}_{0}$ and correlation matrix $\mathrm{M}\left\{\left[\mathrm{x}\left(\mathrm{t}_{0}\right)-\overline{\mathrm{x}}_{0}\right]\left[\mathrm{x}\left(\mathrm{t}_{0}\right)-\overline{\mathrm{x}}_{0}^{\mathrm{T}}\right]\right\}=\mathbf{P}\left(\mathrm{t}_{0}, \mathrm{t}_{0}\right)$. Will assume that the initial state of the system, random influences and measurement noises are mutually uncorrelated for all $t \geq t_{0}$.

The construction of the Kalman filter is reduced to determining the matrix of gain coefficients $\mathbf{K}$, which would provide an optimal estimate (in the sense of the minimum variance of the estimation error) of the state vector.

\section{Discrete Kalman filter}

In a discrete Kalman filter (FK), a continuous dynamic system (1) and measurements (2) correspond to a discrete system recorded in a difference form and discrete measurements

$$
\begin{aligned}
& \overrightarrow{\mathrm{x}}_{\mathrm{k}+1}=\mathbf{F}_{\mathrm{k}} \overrightarrow{\mathrm{x}}_{\mathrm{k}}+\boldsymbol{\Psi}_{\mathrm{k}} \overrightarrow{\mathrm{u}}_{\mathrm{k}}+\boldsymbol{\Gamma}_{\mathrm{k}} \overrightarrow{\mathrm{w}}_{\mathrm{k}} ; \\
& \overrightarrow{\mathrm{y}}_{\mathrm{k}}=\mathbf{H}_{\mathrm{k}} \overrightarrow{\mathrm{x}}_{\mathrm{k}}+\overrightarrow{\mathrm{v}}_{\mathrm{k}},
\end{aligned}
$$

where subscripts «k » and «k $+1 »$ indicate the number of the discrete moment in time $t_{k}$ and $t_{k+1}$; sampling step $\Delta \mathrm{t}=\mathrm{t}_{\mathrm{k}+1}-\mathrm{t}_{\mathrm{k}} ; \mathbf{F}_{\mathrm{k}}=\exp (\mathbf{A} \cdot \Delta \mathrm{t})$ - system transition matrix at time $\mathrm{t}_{\mathrm{k}}$, which we represent approximately in the form of a series expansion

$$
\mathbf{F}_{\mathrm{k}}=\mathbf{I}+\mathbf{F}_{\mathrm{k}} \Delta \mathrm{t}+\frac{1}{2}\left(\mathbf{F}_{\mathrm{k}} \Delta \mathrm{t}\right)^{2}+\ldots
$$

$\boldsymbol{\Gamma}_{\mathrm{k}} \cong \mathbf{F}_{\mathrm{k}} \mathbf{G}_{\mathrm{k}} \Delta \mathrm{t}-$ matrix defining the influence of the input noise vector $\mathrm{w}_{\mathrm{k}-1}$ at the moment $t_{\mathrm{k}}$ At a first approximation $\boldsymbol{\Gamma}_{\mathrm{k}} \approx \mathbf{G}_{\mathrm{k}} \Delta \mathrm{t} ; \boldsymbol{\Psi}_{\mathrm{k}} \cong \mathbf{F}_{\mathrm{k}} \mathbf{B}_{\mathrm{k}} \Delta \mathrm{t}$ - matrix defining the influence of 
the control vector $u_{k-1}$ at the moment $t_{k}$. At a first approximation $\boldsymbol{\Psi}_{\mathrm{k}} \approx \mathbf{B}_{\mathrm{k}} \Delta \mathrm{t} ; \mathbf{I}$ - identity matrix.

Intensity matrices of vector discrete Gaussian noise $\mathbf{w}_{\mathbf{k}}$ system and $\mathbf{v}_{\mathbf{k}}$ measurements are related to the corresponding matrices of a continuous dynamic system by the relations

$$
\mathbf{Q}_{\mathrm{k}}=\frac{\mathbf{Q}\left(\mathrm{t}_{\mathrm{k}}\right)}{\Delta \mathrm{t}}, \quad \mathbf{R}_{\mathrm{k}}=\frac{\mathbf{R}\left(\mathrm{t}_{\mathrm{k}}\right)}{\Delta \mathrm{t}}
$$

Will use the linear discrete Kalman filter algorithm to estimate the state vector :

- set the initial value $\widehat{\mathbf{x}}_{\mathbf{k} 0}$ estimates of the state variable vector, the initial value of the predicted (a priori) error correlation matrix $\breve{\mathbf{P}}_{\mathbf{k} 0}$, noise intensity matrices $\mathbf{Q}_{\mathrm{k}}$ и $\mathbf{R}_{\mathrm{k}}$;

- we get the predicted (apriori) value of the vector of state variables

$$
\breve{\mathbf{x}}_{\mathbf{k}+\mathbf{1}}=\mathbf{F}_{\mathbf{k}} \widehat{\mathbf{x}}_{\mathbf{k}}+\mathbf{B u}_{\mathbf{k}}
$$

- calculate the values of the filter coefficients

$$
\mathbf{K}_{\mathbf{k}}=\breve{\mathbf{P}}_{\mathbf{k}} \mathbf{H}_{\mathbf{k}}^{\mathbf{T}}\left[\mathbf{H}_{\mathbf{k}} \breve{\mathbf{P}}_{\mathbf{k}} \mathbf{H}_{\mathbf{k}}^{\mathbf{T}}+\mathbf{R}_{\mathrm{k}}\right]^{-\mathbf{1}}
$$

- calculate the corrected (posterior) value of the state vector estimate

$$
\widehat{\mathbf{x}}_{\mathbf{k}}=\breve{\mathbf{x}}_{\mathbf{k}}+\mathbf{K}_{\mathbf{k}}\left\lfloor\mathbf{y}_{\mathbf{k}}-\mathbf{H}_{\mathbf{k}} \breve{\mathbf{x}}_{\mathbf{k}}\right\rfloor
$$

- calculate the corrected (a posteriori) correlation matrix of filter errors

$$
\widehat{\mathbf{P}}_{\mathbf{k}}=\left[\mathbf{I}-\mathbf{K}_{\mathbf{k}} \mathbf{H}_{\mathbf{k}}\right] \breve{\mathbf{P}}_{\mathrm{k}}
$$

- calculate the value of the predicted (a priori) error correlation matrix for the new computation step

$$
\breve{\mathbf{P}}_{\mathbf{k}+\mathbf{1}}=\mathbf{F}_{\mathbf{k}} \widehat{\mathbf{P}}_{\mathbf{k}} \mathbf{F}_{\mathbf{k}}^{\mathbf{T}}+\mathbf{Q}_{\mathrm{k}}
$$

- go to a new computation cycle.

To facilitate calculations, you can take the approximate value of the transition matrix $\mathbf{F}=\mathbf{I}+$ A $\Delta \mathrm{t} . \mathrm{n}$ equations is the corrected (posterior) value of the state vector estimate, $\breve{\mathbf{x}}_{\mathbf{k}}-$ the predicted (a priori) value of the state vector estimate, $\mathbf{K}$ - Kalman gain matrix, $\widehat{\mathbf{P}}_{\mathbf{k}}$ - corrected (posterior) correlation matrix of filter errors, $\breve{\mathbf{P}}_{\mathbf{k}}$ - predicted (a priori) correlation matrix of 
filter errors, R- observation (measurement) noise intensity matrix $\mathbf{v}, \mathbf{Q}_{\mathrm{k}}-$ input disturbance intensity matrix $\mathbf{w}$. Initial matrix $\mathbf{P}_{\mathbf{k}}$ usually contains diagonal elements corresponding to the initial error variances of the corresponding state variables.

It is necessary to use a Kalman filter to remove noise from the useful signal.

\section{Kalman averaging filter}

\subsection{Filter algorithm}

The Kalman averaging filter algorithm can be represented as follows.

Let us assume that the errors of the accelerometers $\Delta \mathrm{a}_{\mathrm{i}}$ and gyros $\omega_{\mathrm{di}}$

$$
\Delta \mathrm{a}_{\mathrm{i}}=\mu_{\mathrm{i}}+\sigma_{\mathrm{ai}} \mathrm{w}_{\mathrm{i} 1}, \quad \omega_{\mathrm{di}}=\varepsilon_{\mathrm{i}}+\sigma_{\omega \mathrm{i}} \mathrm{w}_{\mathrm{i} 2}, \quad \mathrm{i}=\mathrm{x}, \mathrm{y}, \mathrm{z} .
$$

have a systematic $\mu_{\mathrm{i}}, \varepsilon_{\mathrm{i}}$ and random components $\sigma_{\mathrm{ai}} \mathrm{W}_{\mathrm{i} 1} ; \sigma_{\omega \mathrm{i}} \mathrm{W}_{\mathrm{i} 2}$.

Let's write signals with systematic errors

$$
\omega_{i}^{c}=\omega_{i}+\varepsilon_{i}, \quad a_{i}^{c}=a_{i}+\mu_{i}, \quad i=x, y, z
$$

We take these signals as state variables and compose a state variables vector

$$
\vec{x}=\left(\omega_{x}^{c}, \omega_{y}^{c}, \omega_{z}^{c}, a_{x}^{c}, a_{y}^{c}, a_{z}^{c}\right)^{T}
$$

We write the equation of dynamics (state) in the form

$$
\overrightarrow{\mathrm{x}}_{\mathrm{k}+1}=\overrightarrow{\mathrm{x}}_{\mathrm{k}}+\overrightarrow{\mathrm{w}}_{\mathrm{k}}
$$

It is seen from this equation that the transition matrix $\mathbf{F}_{\mathrm{k}}$ is the identity matrix $\mathbf{I}$.

Let us assume that the random components $\sigma_{\omega \mathrm{i}} \cdot \mathrm{w}_{\mathrm{i} 1}, \sigma_{\mathrm{ai}} \cdot \mathrm{w}_{\mathrm{i} 2}, \quad \mathrm{i}=\mathrm{x}, \mathrm{y}, \mathrm{z}$ are white noise, $\sigma_{\omega \mathrm{i}}$ and $\sigma_{\mathrm{ai}}$ - root-mean-square errors of signals from angular rate sensors and accelerometers, $\overrightarrow{\mathrm{w}}=\left[\begin{array}{ll}\mathrm{w}_{\mathrm{i} 1} & \mathrm{w}_{\mathrm{i} 2}\end{array}\right]^{\mathrm{T}}=\mathrm{w}_{(6,1)}$ - generating "white noise" of unit intensity. Will write the equation of measurements in the form

$$
\overrightarrow{\mathrm{y}}_{\mathrm{k}}=\mathbf{H}_{\mathrm{k}} \overrightarrow{\mathrm{x}}_{\mathrm{k}}+\overrightarrow{\mathrm{v}}_{\mathrm{k}} \text {. }
$$


Measurement vector $\vec{y}_{k}=\left[\omega_{x}^{c}, \omega_{y}^{c}, \omega_{z}^{c}, a_{x}^{c}, a_{y}^{c}, a_{z}^{c}\right]^{T}$. Hence the measurement matrix will be $H=\operatorname{diag}\left(\left[\begin{array}{llllll}1 & 1 & 1 & 1 & 1 & 1\end{array}\right]\right)$, measurement noise $\overrightarrow{\mathrm{v}}_{\mathrm{k}}=\overrightarrow{\mathrm{w}}_{\mathrm{k}}, \operatorname{diag}\left(\mathrm{f}_{1} \mathrm{f}_{2} \quad \ldots \quad \mathrm{f}_{\mathrm{n}}\right)-$ shorthand notation for a diagonal matrix with $\mathrm{f}_{1} \quad \mathrm{f}_{2} \quad \ldots \quad \mathrm{f}_{\mathrm{n}}$ - elements of the main diagonal. Perturbation matrix

$$
\mathrm{Q}=\mathrm{kQ} \cdot \operatorname{diag}\left(\sigma_{\omega \mathrm{x}}^{2} \sigma_{\omega \mathrm{y}}^{2} \sigma_{\omega \mathrm{z}}^{2} \quad \sigma_{\mathrm{ax}}^{2} \sigma_{\mathrm{ay}}^{2} \sigma_{\mathrm{az}}^{2}\right)
$$

Measurement matrix

$$
\mathrm{R}=\mathrm{kR} \cdot \operatorname{diag}\left(\sigma_{\omega \mathrm{x}}^{2} \sigma_{\omega \mathrm{y}}^{2} \sigma_{\omega \mathrm{z}}^{2} \quad \sigma_{\mathrm{ax}}^{2} \sigma_{\mathrm{ay}}^{2} \sigma_{\mathrm{az}}^{2}\right)
$$

Here $\mathrm{kQ}$ и $\mathrm{kR}$ - tuning coefficients.

Than we obtain a forward averaging KF by applying the discrete linear Kalman filter algorithm.

\subsection{A forward averaging filter simulation}

Figure 2 shows the gyro signals and the results of their averaging by the forward Kalman filter. Will put for simulation $\mathrm{kR}=1 \mathrm{e} 3, \mathrm{kQ}=0,1$. The graphs show the transient filtration process. Increasing $\mathrm{kR}$ helps to increase noise smoothing (increasing std), but increases the duration of the transient. Increasing $\mathrm{kQ}$ helps to reduce the duration of the transient, but worsens smoothing. It is obvious that the transient of the filter (in fig. 2 it takes $3 \mathrm{sec}$ ) should be excluded from further work.

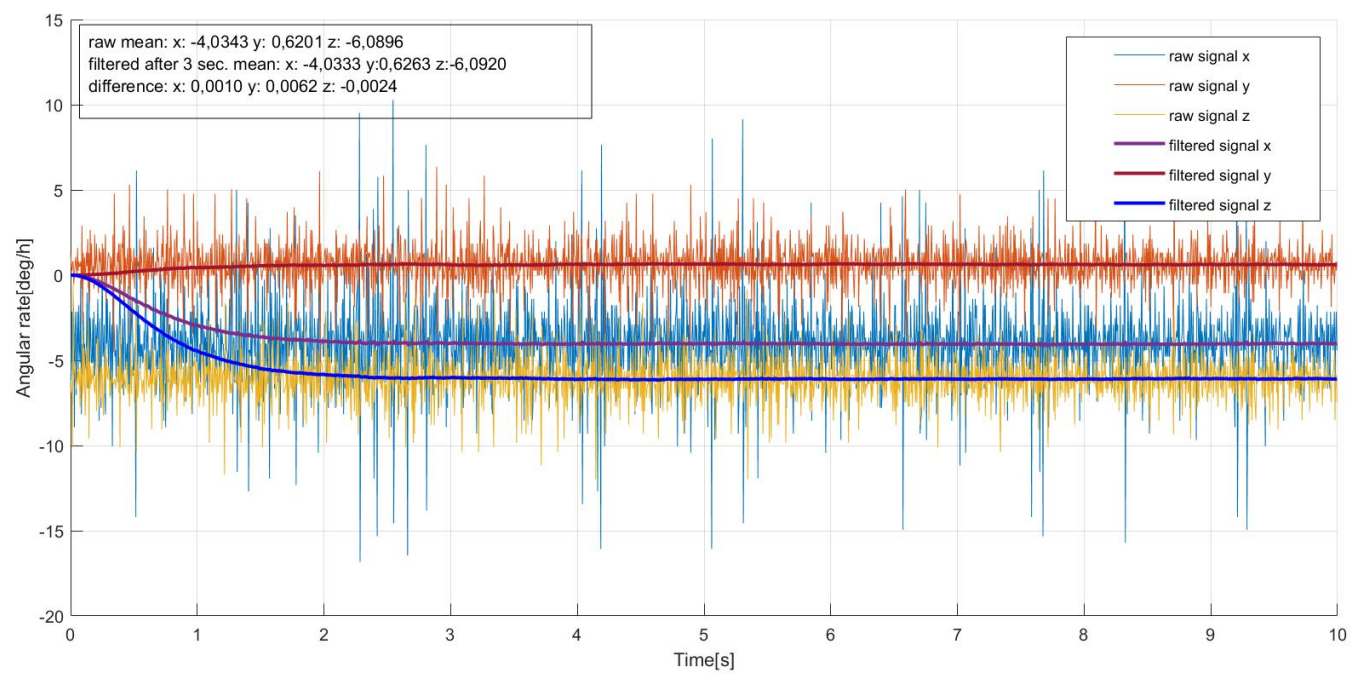

Figer 2. Gyro signal averaging (receiving useful signal) using the forward_Kalman filter 
When simulating in each numerical experiment, the noise generator creates a sequence that changes with each run. Therefore, the conclusion should be drawn from a series of experiments (statistical test method). The results for 3 numerical experiments are shown in Table 1.

Table 1. Averaging simulation results for a fixed base $. \sigma_{\mathrm{n}}=27 \mathrm{deg} / \mathrm{hr}$, signal duration tosr $=30 \mathrm{sec}$, sampling step $\mathrm{h}=0,01 \mathrm{sec}$

\begin{tabular}{|c|c|c|c|c|c|c|c|c|c|c|c|c|c|}
\hline & \multicolumn{9}{|c|}{ Gyro signals, deg/hr } \\
\hline & $\begin{array}{c}\text { Start } \\
1\end{array}$ & $\begin{array}{c}\text { Start } \\
2\end{array}$ & Start & 3 & aver & Start & Start \\
1 & 2 & Start & 3 & aver & Start 1 & Start 2 & Start 3 & aver & \\
\hline original & $-6,734$ & $-6,734$ & $-6,734$ & $-6,734$ & 7,035 & 7,035 & 7,035 & 7,035 & 11,825 & 11,825 & 11,825 & 11,825 & \\
\hline simple average & $-6,806$ & $-6,407$ & $-6,469$ & $-6,561$ & 7,268 & 6,907 & 7,331 & 7,168 & 10,840 & 11,672 & 11,342 & 11,285 & \\
\hline forward FK & $-6,734$ & $-6,734$ & $-6,734$ & $-6,734$ & 7,035 & 7,035 & 7,035 & 7,035 & 11,825 & 11,825 & 11,825 & 11,825 & \\
\hline $\begin{array}{c}\text { simple average } \\
\text { error }\end{array}$ & & & 0,173 & & & 0,134 & & & $-0,540$ & 0,336 \\
\hline forward FK error & & & $-2,7 e-5$ & & & $5 e-5$ & & & $3,3 \mathrm{e}-5$ & $3,8 \mathrm{e}-5$ \\
\hline
\end{tabular}

The accuracy increasing can be achieved by adjusting the FK (changing the matrices P, Q, R ).

Simulation shows that to improve accuracy, we can reduce the coefficient $\mathrm{kR}$, leaving the coefficient $\mathrm{kQ}=1$ (Fig.3).

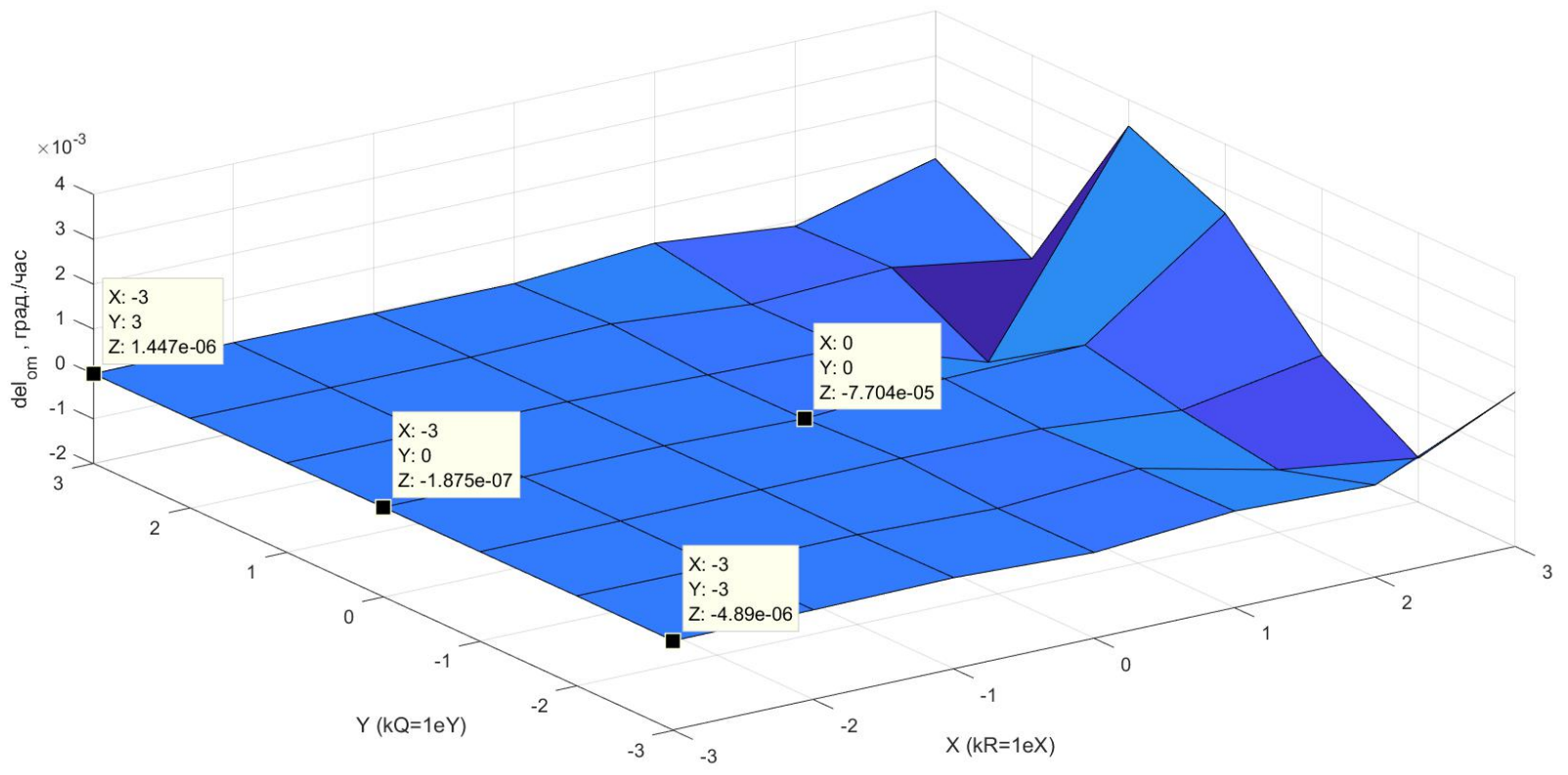

Figer.3. Error surface when tuning coefficients 
As can be seen from Table 1, smoothing by the Kalman filter is performed much better than when using a simple average.

\section{Averaging Kalman filter with backward motion (backward filter)}

The noise reduction task can be accomplished by applying the averaging Kalman filter (forward filter), as well as the Kalman filter in reverse time (backward filter) $[9,10,11,12$, 13]. Expressions of the suboptimal backward filter practically coincide with the equations of the forward Kalman filter [9]. This allows the standard Kalman filter software to be used for a robust numerical implementation of the inverse backward filter. The computational procedures are as follows.

\subsection{Backward filter start point initialization}

$$
\widetilde{\mathrm{x}}_{\mathrm{bN}}^{-}=0, \quad \mathrm{P}_{\mathrm{bN}}^{-}=\mathrm{m} \cdot \mathrm{I}
$$

( $\mathrm{m}$ - big number, index $\mathrm{b}$ means backward, $\mathrm{N}$ - end number of counts of forward stroke, $\mathrm{I}-$ dentity matrix).

In this case, a significant reverse flow transient is likely. To reduce it, you can take the final value of the evaluation of the forward stroke as the starting point of the backwarde stroke $\widetilde{\mathrm{x}}_{\mathrm{bN}}^{-}=\widetilde{\mathrm{x}}_{\mathrm{N}}^{-}$, and the final value of the covariance matrix of the forward run errors is taken as the starting covariance matrix of the backward run $\mathrm{P}_{\mathrm{bN}}^{-}=\mathrm{P}_{\mathrm{N}}^{-}$.

4.2 Measurement processing stage (keeping the designations [9])

$$
\begin{aligned}
& \tilde{\mathrm{x}}_{\mathrm{bj}}^{+}=\tilde{\mathrm{x}}_{\mathrm{bj}}^{-}+\mathrm{K}_{\mathrm{bj}}\left[\mathrm{z}_{\mathrm{j}}-\mathrm{H}_{\mathrm{j}} \tilde{\mathrm{x}}_{\mathrm{bj}}^{-}\right], \\
& \mathrm{P}_{\mathrm{bj}}^{+}=\left[\mathrm{I}-\mathrm{K}_{\mathrm{bj}} \mathrm{H}_{\mathrm{j}}\right] \mathrm{P}_{\mathrm{bj}}^{-}, \\
& \mathrm{K}_{\mathrm{bj}}=\mathrm{P}_{\mathrm{bj}}^{-} \mathrm{H}_{\mathrm{j}}^{\mathrm{T}}\left[\mathrm{H}_{\mathrm{j}} \mathrm{P}_{\mathrm{bj}}^{-} \mathrm{H}_{\mathrm{j}}^{\mathrm{T}}+\mathrm{R}_{\mathrm{j}}\right]^{-1} .
\end{aligned}
$$

These ratios exactly coincide with the ratios of the correction stage of the forward Kalman filter. 


\subsection{Forecast stage}

$$
\begin{aligned}
& \tilde{\mathrm{x}}_{\mathrm{bj}-1}^{-}=\Phi_{\mathrm{j}, \mathrm{j}-1}^{-1} \tilde{\mathrm{x}}_{\mathrm{bj}}^{+}, \\
& \mathrm{P}_{\mathrm{bj}}^{-}=\Phi_{\mathrm{j}, \mathrm{j}-1}^{-1}\left[\mathrm{P}_{\mathrm{bj}}^{+}+\mathrm{Q}_{\mathrm{j}-1}\right] \Phi_{\mathrm{j}, \mathrm{j}-1}^{-\mathrm{T}}, \\
& \Phi_{\mathrm{j}, \mathrm{j}-1}^{-\mathrm{T}}=\left[\Phi_{\mathrm{j}, \mathrm{j}-1}^{-1}\right]^{\mathrm{T}} .
\end{aligned}
$$

$\Phi^{-1} \equiv F^{-1}$ - the inverse of the forward filter transition matrix.

For averaging filter $\Phi=\mathrm{I}, \mathrm{Q}=\mathrm{R}$.

\subsection{Backward filter simulation}

The simulation results of the backward_Kalman filter are shown in Fig. 4, 5
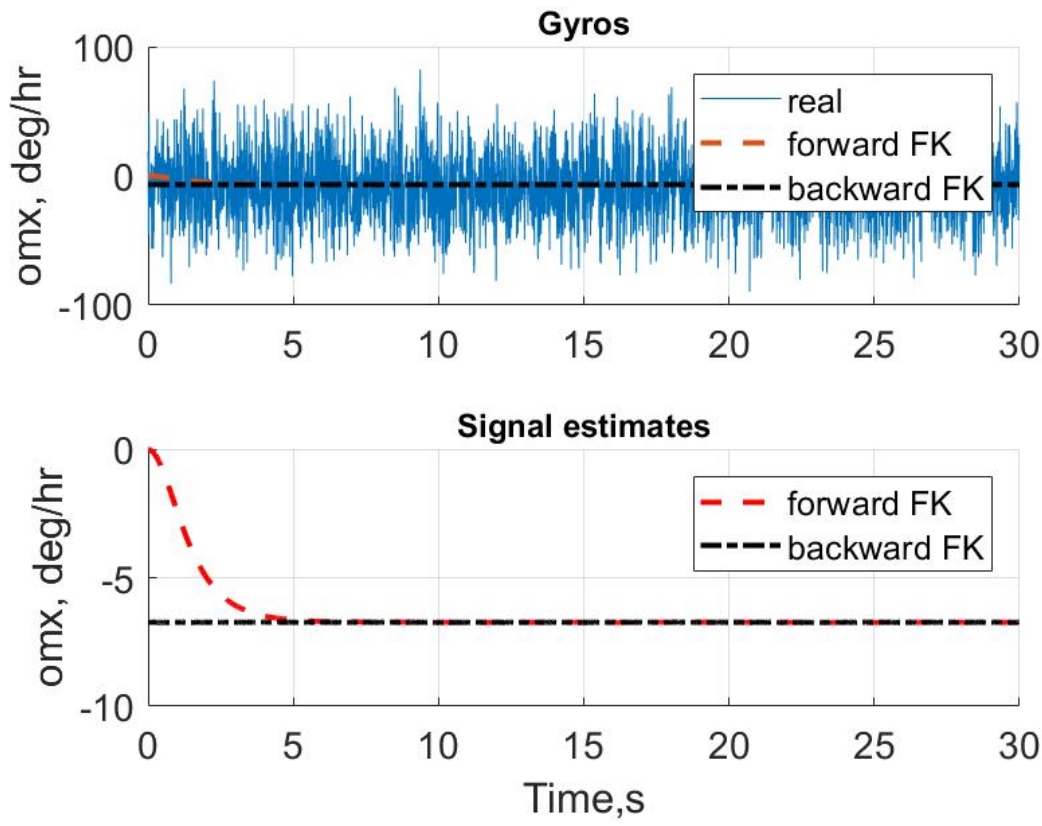

Figer.4. Gyro signal and systematic component estimates

forward and backward Kalman filters.

The advantage of an inverse filter is that it (as opposed to a forward filter) operates under precise initial conditions (initial values of the variables $\widetilde{\mathrm{x}}_{\mathrm{bN}}^{-}=\widetilde{\mathrm{x}}_{\mathrm{N}}^{-}$and the error covariance matrix $\mathrm{P}_{\mathrm{bN}}^{-}=\mathrm{P}_{\mathrm{N}}^{-}$). If in the evaluation of the forward filter it is necessary to exclude the transient process, then in the evaluation of the inverse filter there is practically no transient. 


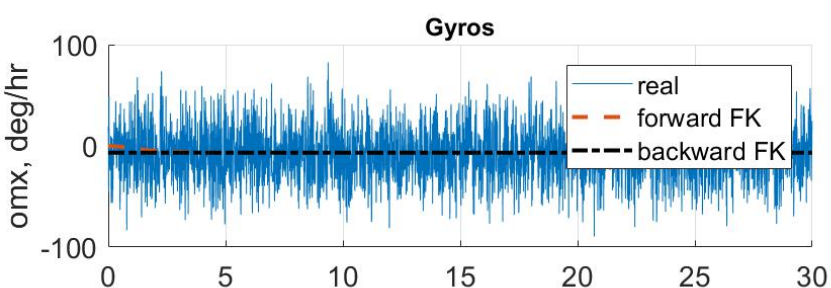

Noise $(\mathrm{std})$ slom $=27$ (deg./hr) original omsys $(1)=-6.73422(\mathrm{deg} . / \mathrm{hr})$ simple average, $\mathrm{sx3g}=-7.27959$ (deg./hr) dpx $=-0.545371$ (deg./hr) forward FK, sx1g = -6.73149 (deg./hr) $\quad \mathrm{dfx}=0.00273344$ (deg./hr) backward FK, sbx1g = -6.73532 (deg./hr) dbx = -0.00109654 (deg./hr)

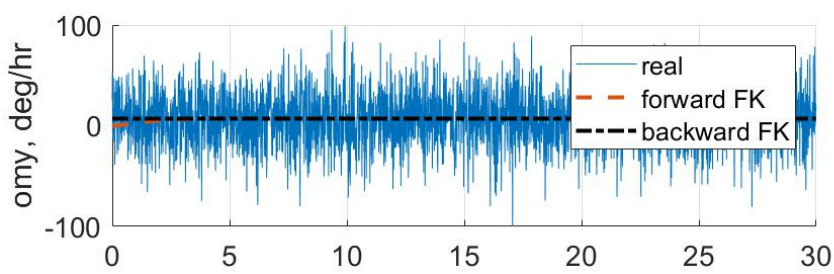

original omsys $(2)=7.03473$ (deg./hr) simple average, sy3g $=7.14512(\mathrm{deg} . / \mathrm{hr}) \mathrm{dpy}=0.110396(\mathrm{deg} . / \mathrm{hr})$ forward FK, sy1g = 7.03138 (deg./hr) dfy $=-0.00335078$ (deg./hr) $\mathrm{FK}$, sby $1 \mathrm{~g}=7.03523(\mathrm{deg} . / \mathrm{hr})$ $\mathrm{dby}=0.000502031(\mathrm{deg} . / \mathrm{hr})$

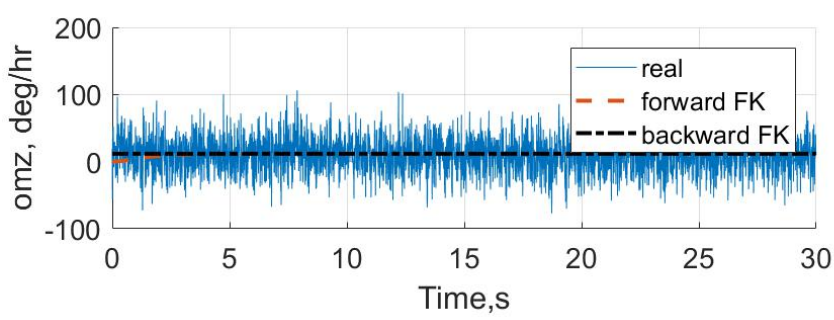

original omsys $(3)=11.8245(\mathrm{deg} . / \mathrm{hr})$ simple average, $\mathrm{sz3g}=10.5433(\mathrm{deg} . / \mathrm{hr}) \mathrm{dpz}=-1.28128(\mathrm{deg} . / \mathrm{hr})$ forward FK, sz1g = $11.8182($ deg./hr) $\quad \mathrm{dfz}=-0.00639218($ deg. $/ \mathrm{hr})$ backward FK, sbz1g = 11.8248 (deg./hr) dbz $=0.000246414$ (deg./hr)

Figer.5. Characteristics of estimates by the forward and backward Kalman filter

Shown in Fig. 5. errors correspond to the average error values for the last $25 \mathrm{sec}$ of the process (forward filter transient excluded). As can be seen from Fig. 5, the errors of the forward filter are several times less than the errors of averaging by the arithmetic mean (simple mean), and the errors of the inverse filter are several times less than the errors of the forward filter.

\section{Natural experiment}

Let us compare the results of applying the forward and backward averaging filters with an autonomous initial alignment (Table 2) according to the universal algorithm given in [2]. A measuring unit with the following characteristics was used in the experiments:

- gyro zero instability - 0,01 deg/hr;

- instability of gyro scale factor - 50 ppm (5e-5);

- accelerometers zero instability - $2 \mathrm{e}-3 \mathrm{~m} / \mathrm{s}^{2}$;

- instability of accelerometers scale factor - 200 ppm (2 e-4).

The block noise characteristics are shown in Fig. 1. 
The alignment results (Tab. 2) were obtained when recording for $30 \mathrm{~s}$ and setting the direct filter $\mathrm{kQf}=0.01, \mathrm{kRf}=1 \mathrm{e} 3$ and reverse filter $\mathrm{kQb}=1, \mathrm{kRb}=1 \mathrm{e}-2$.

Table 2. Results of an autonomous alignment by heading when using forward and backward averaging Kalman filters

\begin{tabular}{|c|c|c|c|c|c|}
\hline & \multicolumn{2}{|c|}{ Backward filter heading angle } & \multicolumn{2}{c|}{ Forward filter heading angle } \\
\hline $\begin{array}{c}\text { № } \\
\text { of test. }\end{array}$ & True, deg & filter, deg & $\begin{array}{c}\text { Error, } \\
\text { angular } \\
\text { minute }\end{array}$ & $\begin{array}{c}\text { filter., } \\
\text { deg }\end{array}$ & $\begin{array}{c}\text { Error, angular } \\
\text { minute }\end{array}$ \\
\hline 1 & 356,466 & 356,508 & 2,54 & 356,542 & 4,592 \\
\hline 2 & 356,466 & 356,515 & 2,936 & 356,518 & 3,158 \\
\hline 3 & 356,466 & 356,472 & 0,38 & 356,471 & 0,302 \\
\hline 4 & 356,466 & 356,449 & $-1,018$ & 356,497 & 1,874 \\
\hline 5 & 356,466 & 356,458 & $-0,472$ & 356,474 & 0,506 \\
\hline 6 & 356,466 & 356,457 & $-0,514$ & 356,435 & $-1,822$ \\
\hline 7 & 358,415 & 358,419 & 0,218 & 358,383 & $-1,906$ \\
\hline 8 & 358,415 & 358,453 & 2,276 & 358,403 & $-0,73$ \\
\hline & rms 8 & & 1,657 & rms 8 & 2,302 \\
\hline
\end{tabular}

The backward filter worked with a sample of $30 \mathrm{~s}$ (like the forward filter) after the forward filter. Such a sequence lengthens the alignment process not much, since the processing speed is much higher than the measuring speed. The time interval of the signal processed by the backward filter is larger by the time of the transient process of the forawrd FK. With the correct initialization of the backward filter $(\mathrm{Pb0}=\mathrm{P})$, there is practically no transient in it (Fig. 4). The backward filter is applied during the heading angle estimation phase because the roll and pitch estimation is performed reasonably well without an backward filter. For the final estimate, the root mean square errors (rms) from 8 experiments were used.

\section{Conclusions}

Simulation the proposed algorithms of forward and backward averaging Kalman filters has shown that the use of averaging Kalman filters significantly improves the filtering of signals from noise in comparison with averaging (filtering) by the arithmetic mean. A significant positive result is achieved with the sequential application of 
forward and backward averaging filters.

\section{Reference}

[1] D.H. Titterton and J.L. Weston, Strapdown Inertial Navigation Technology, 2nd ed. London, UK: IET, 2004, - 558 p.

[2] O. Zbrutsky, S. Lakoza, V. Meleshko, R. Chornomorets. Autonomous alignment of strapdown inertial navigation system on oscillating base. 2019 IEEE 2nd Ukraine Conference on Electrical and Computer Engineering (UKRCON). doi:10.1109/ukrcon.2019.8879993

[3] A.A. Leonets. Systemic problems of strapdown inertial navigation systems. Part 1. Complex solution: monograph / AA Leonets. - K .: Alpha Advertising, 2018 .- 732 p.(in Russian)

[4] Greg Welch , Gary Bishop . An Introduction to the Kalman Filter. University of North Carolina, SIGGRAPH 2001, Los Angeles.

[5] Mohinder S.Grewal, Angus Andrews. Kalman filtering: theory and practice using MATLAB. California State University, Fullerton, January 2001, DOI: $10.1002 / 9780470377819$

[6] A.N.Yakovlev. Introduction to Wavelet Transforms: A Tutorial. -Novosibirsk: Publishing house of NSTU, 2003. - 104 p. (in Russian)

[7] S.V. Moskovsky, A.N. Sergeev, N.A. Lalina. Signal cleaning from noise using wavelet transform // Universum: Technical sciences: electron. scientific. Joun. 2015. No. 2 (15).(in Russian).http://7universum.com/ru/tech/archive/item/1958

[8] El-Sheimy N., Nassar S., Noureldin A. Wavelet de-noising for IMU alignment. Aerosp. Electron. Syst. Mag. 2004, 19, 32-39.

[9] V.O. Brown, V.P. Dolgushin, V.N. Loza, I.V. Pampukha. Investigation of the possibilities and characteristics of methods for reducing the noise level in signal processing based on the use of wavelet technology. Journal of Radio Electronics (electronic journal), No. 7, 2014. P.P.

[10] A.A. Golovan, N.A. Parusnikov Mathematical foundations of navigation systems. Part 2. Application of optimal estimation methods to navigation problems. - M .: MAKS Press, 2012 .-- 172 p.(in Russian)

[11] P.S. Maybeck. Stochastic models, estimation and control. New York: Academic Press. 1979. 
[12] Houzeng Han, Jian Wang, Mingyi Du. A Fast SINS Initial Alignment Method Based on RTS Forward and Backward Resolution. -Hindawi. Journal of Sensors, Volume 2017, Article ID 7161858, 11 pages. https://doi.org/10.1155/2017/7161858

[13] Haiying Liu, Xin Jiang, Guangen Gao. Application of reverse processing in SINS initial alignment and SINS/GPS integrated navigation. Aircraft Engineering and Aerospace Technology, https://doi.org/10.1108/AEAT-11-2016-0191

[14] H.Han, J.Wang, M.Du. A fast SINS initial alignment method based on RTS forward and backward resolution. Hindawi journal of sensors. Volume 2017, article, id 7161858, 11 p. doi.org/10.1155/2017/7161858 